livraisons

d'Histoire

de l'Architecture

\section{Livraisons de l'histoire de l'architecture}

32 | 2016

Les représentations de l'architecture

\title{
Entre Las Luces y el Antiguo : notes et précisions sur le dessin d'architecture à l'Académie de Saint Ferdinand (1752-1808)
}

Entre las luces y el Antiguo: notes and remarks about the architectural drawing at the Academy of fine arts of Saint Ferdinand (1752-1808)

Entre las luces y el antiguo. Anmerkungen zur Architekturzeichnung an der Academia de San Fernando (1752-1808)

\section{Adrián Almoguera}

\section{OpenEdition}

Journals

Édition électronique

URL : http://journals.openedition.org/lha/635

DOI : 10.4000/lha.635

ISSN : 1960-5994

Éditeur

Association Livraisons d'histoire de l'architecture - LHA

Édition imprimée

Date de publication : 31 décembre 2016

Pagination : $37-51$

ISSN : 1627-4970

Référence électronique

Adrián Almoguera, «Entre Las Luces y el Antiguo : notes et précisions sur le dessin d'architecture à

I'Académie de Saint Ferdinand (1752-1808) ", Livraisons de l'histoire de l'architecture [En ligne],

32 | 2016, mis en ligne le 31 décembre 2018, consulté le 01 mai 2019. URL : http://

journals.openedition.org//ha/635; DOI : 10.4000/lha.635 


\title{
ENTRE LAS LUCES Y EL ANTIGUO: NOTES ET PRÉCISIONS SUR LE DESSIN D'ARCHITECTURE À L'ACADÉMIE DE SAINT-FERDINAND (1752-1808)
}

\begin{abstract}
À l'aube des Lumières, l'Espagne n'avait pas encore adopté le modèle des académies d'art qui fleurissait dans le reste de l'Europe. À partir de 1736, le chantier du Palacio Nuevo, qui se prolonge pendant plus de trente ans, mit en évidence le manque de préparation des architectes nationaux face à un projet d'une telle envergure. Pour combler ce besoin, le pouvoir royal encouragea la fondation d'une académie à Madrid, l'Académie de Beaux-Arts de Saint-Ferdinand, qui ouvrit officiellement ses portes en $1752^{1}$. Inspirée des modèles parisien et romain afin d'institutionnaliser (et contrôler) l'enseignement artistique à échelle nationale, l'académie de Madrid a fondé sa pédagogie sur un principe supposé d'Aequa potestas parmi les trois "arts majeurs " ${ }^{2}$. Mais ce principe ne fut respecté que sur le papier, puisque dès les premières années, l'architecture constitua l'objet principal du débat artistique qui avait lieu dans ses murs. Ce prestige croissant provient du sentiment de supériorité exprimé par les architectes et a provoqué de nombreuses controverses entre architectes, et peintres et sculpteurs. Aux yeux du roi, c'était dans les salles d'architecture de San Fernando que l'on formait les futurs professionnels censés transformer l'image architecturale du nouvel État bourbonien. Par ailleurs, les mécanismes académiques comme la Commission d'architecture, créé en 1786, permettaient de contrôler le devenir de toute sorte de projets d'architecture religieuse ou publique sur l'ensemble du territoire espagnol ${ }^{3}$. À l'origine de ce processus de légitimation institutionnelle et artistique de l'architecture académique, se trouve le dessin d'architecture, un outil de travail qui s'est rapidement transformé en objet artistique autonome. Dans le temps entre la création de l'Académie et l'effondrement provoqué par la Guerre d'Indépendance, les dessins d'architecture de San Fernando furent la justification de la nécessité du projet de rénovation politique et culturel mené par la royauté pour assurer le progrès de la nation à travers l'enseignement de l'art de bâtir.
\end{abstract}

1. Sur l'histoire de l'académie voir notamment Claude Bédat, L'Académie de Beaux-Arts de Madrid: 1744-1808, Toulouse, Toulouse-Le Mirail, 1973, 478 p.

2. L'étude comparative la plus complète sur l'activité des trois académies se trouve dans Basile Baudez, Architecture et tradition académique, Rennes, PUR, 2012, 390 p.

3. Concernant le fonctionnement de la Commission d'architecture, voir Jose Enrique García Melero, "Arquitectura y burocracia : el proceso del proyecto en la Comisión de Arquitectura de la Academia 1786-1808», Espacio, Tiempo y Forma, t. IV, 1991, p. 283-348. 


\section{Le dessin d'architecture dans l'académie de San Fernando: de l'outil de travail à la référence esthétique}

Dès 1757 , les premiers statuts académiques reflètent la priorité donnée à la création de collections artistiques servant de modèles pour les jeunes étudiants ${ }^{4}$. Aux collections de plâtres pour les sculpteurs, furent ajoutés les gravures et tableaux pour les peintres. Dans le même temps, les autorités académiques constituèrent une riche bibliothèque contenant les principaux recueils et publications de référence européens ${ }^{5}$. Pour les architectes fut d'abord constituée une collection de dessins d'architecture à partir des dons des enseignants, collection qui fut rapidement augmentée par les envois des pensionnaires qui rapportaient à Madrid les dernières nouveautés européennes. Ces envois étaient des références très recherchées par les jeunes élèves qui les accrochaient aux murs pour les observer et s'exercer à les copier jour et nuit à la bougie, procédure qui a provoqué la perte et la dégradation de nombreux exemples de grande valeur ${ }^{6}$. La conception du dessin d'architecture académique comme objet esthétique en soi, capable de modifier la pensée architecturale des jeunes élèves, mena les académiciens à proposer des espaces d'exposition spécifiques dans l'académie de Madrid. Par deux fois, le programme des concours d'architecture a proposé "un bâtiment pour les académies d'art "; chaque fois, le jury a insisté sur la nécessité de créer des "salles pour les dessins " (1756), et même "une galerie pour [exposer] les plans d'architecture" (1796) ${ }^{7}$. De cette façon, les architectes de San Fernando ont appris les fondements de leur métier : en dessinant côte à côte dans des salles aux murs couverts de dessins d'architecture encadrés et exposés à la vue de tous (ill. 1). L'étude de la rotation et de la substitution de ces dessins, présentés aux élèves comme les modèles les plus novateurs, révèle une évolution typologique et stylistique qui se reflète aussi dans les choix de composition et des techniques des rendus dans les concours académiques ${ }^{8}$. Pendant les premières décennies d'enseignement, les dessins exposés étaient des exemples d'architecture baroque à l'italienne en petit et moyen format, principalement réalisés par les enseignants responsables du chantier du Palais Royal et par leurs élèves. Vers la fin du siècle, au contraire, les projets novateurs d'architecture civile proposés par les archi-

4. Sur les premiers statuts de 1757 et leur rapport à la création de collections de dessins et d'un premier cours méthodologique d'architecture voir Archives de la Real Academia de Bellas artes de San Fernando de Madrid (désormais ASF) 1-18-9.

5. Nous renvoyons à l'étude de Claude Bédat, "La biblioteca de la Real Academia de San Fernando en 1793 ", Academia, vol. 25 et 26, 1968, qui malheureusement ne considère pas les achats fondamentaux faits entre 1793 et 1808 .

6. Les dessins sont cités dans les inventaires des dessins raccrochés aux murs. Les termes employés - " muy ahumado ", " muy rajado, o muy roto " - permettent de voir qu'ils y demeuraient longtemps. ASF 2-57-4 et 2-57-5.

7. Les programmes des concours ont été reproduits dans Delfín Rodríguez, Hacia una nueva idea de la Arquitectura: Premios generales de Arquitectura de la Real Academia de Bellas artes de San Fernando 1753-1831, Madrid, RABASF, 1992, 198 p.

8. Pour les inventaires de ces dessins exposés dans les salles d'architecture voir ASF 2-57-4 et 2-57-5. 


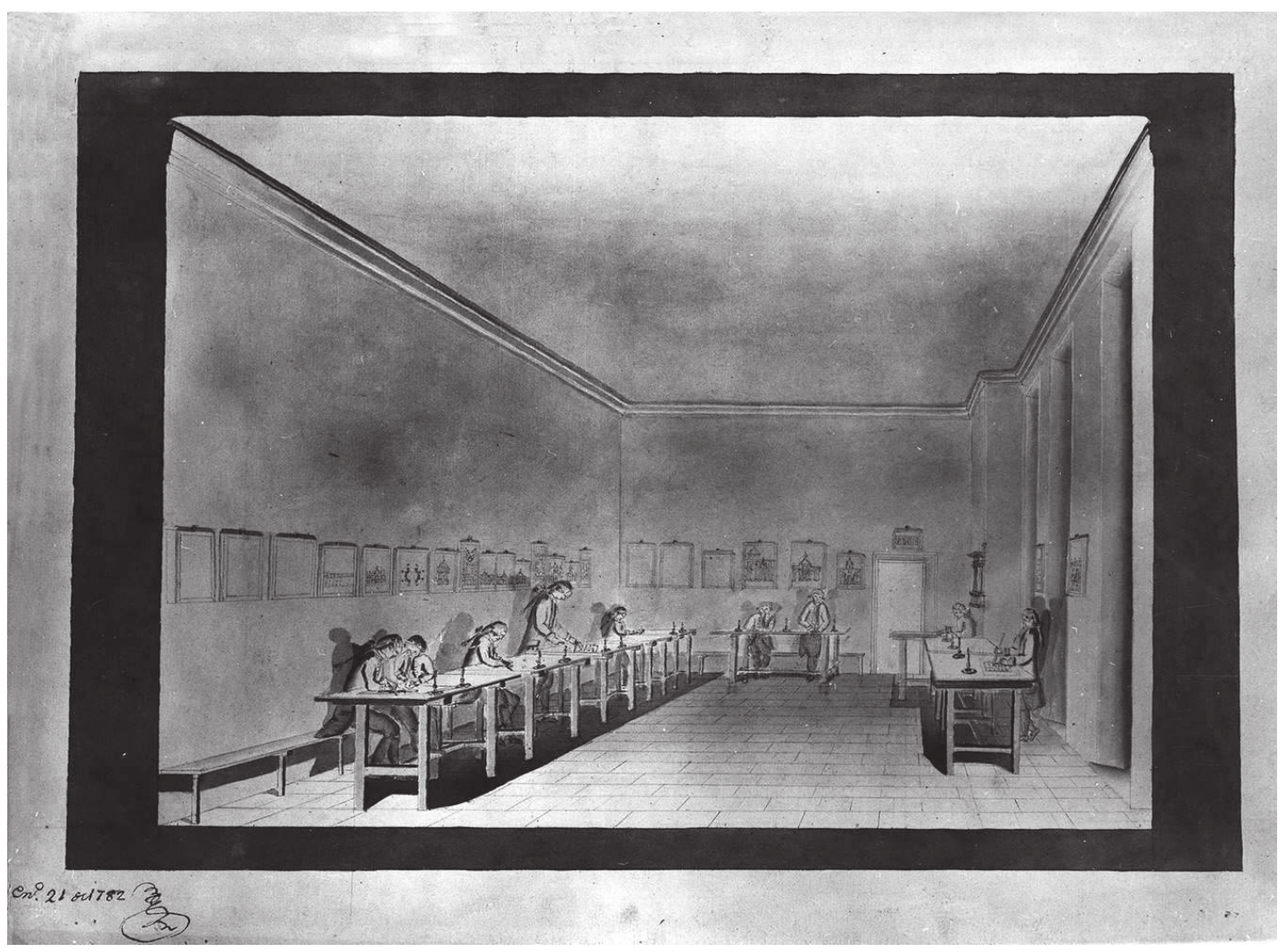

Ill. 1 : José de Toralla, La salle d'architecture de l'Académie de Saint-Ferdinand, 1782, $30 \times 35 \mathrm{~cm}$, crayon, encre de Chine, lavis brun. (C) Madrid, Real Academia de Bellas Artes de San Fernando, P-2298.

tectes les mieux formés dans la Rome internationale s'imposent. La prépondérance des dessins signés par Silvestre Pérez ou Isidro González Velázquez sur les murs de l'académie autour de 1800 explique en grande partie la transformation technique et stylistique opérée dans le dessin académique de cette époque. Ce changement a des conséquences manifestes surtout dans la première décennie du XIX ${ }^{\mathrm{e}}$ siècle, quand les thèmes, les types et les formes annoncent à Madrid une nouvelle idée de l'architecture.

À San Fernando, la copie demeure la base de l'apprentissage, qu'elle soit réalisée d'après les modèles incontournables des gravures conservées dans la bibliothèque académique, ou d'après les dessins exposés dans les salles, encadrés ou accrochés aux murs ${ }^{9}$. Dès leur arrivée, les jeunes étudiants copiaient inlassablement, en s'appuyant sur une méthode assez productive dans les premiers temps de l'académie, mais qui devint très lourde et certainement inintéressante dès les années 1780 ; en étudiant de près la plupart des dessins d'architecture datés avant cette date, on reconnaît facilement la méthode de copie analytique des élèves (ill. 2).

9. Outre les dessins exposés dans les salles de manière prolongée, les rendus des concours d'architecture et les envois des pensionnaires faisaient l'objet d'expositions publiques où les élèves travaillaient sur leurs copies et le public madrilène, attiré par la presse, venait admirer la production de l'académie. Sur ces expositions voir les Actes de l'assemblée générale, ainsi que ASF 1-55-2. 


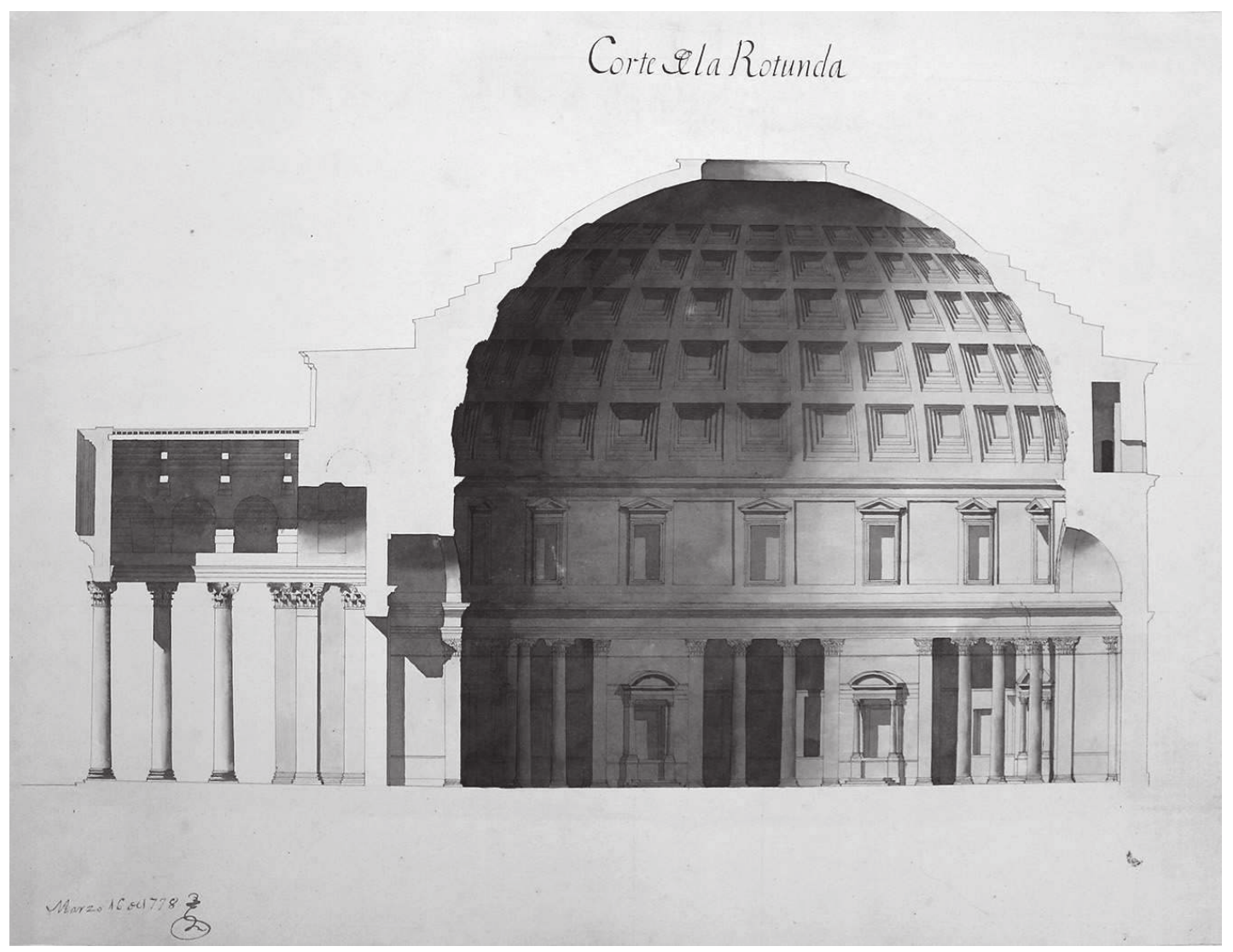

Ill. 2 : Ignacio Haan, Vue du Panthéon de Rome (coupe longitudinale), Ayuda de Costa pour la deuxième classe d'architecture de 1778, 64,6 × 47,8 cm, encre de Chine, lavis gris et rosetrois dessins à l'encre de Chine, lavis gris et rose. (C) Madrid, Real Academia de Bellas Artes de San Fernando, A-492 - A-494.

Avec la permission de leurs professeurs, ces étudiants se sont toujours consacrés à une soigneuse sélection de différents éléments stylistiques et de composition empruntés aux sources graphiques et théoriques mises à disposition par l'académie. Telle fut l'enracinement de cette procédure que, contrairement à ce que l'assemblée recommandait dès 1757, on relève, dans l'instruction donnée aux pensionnaires à Rome en 1791, l'obligation de "mesurer et relever les ruines antiques et toute sorte de fabrique d'après nature, et surtout pas à partir d'autres dessins ou estampes ", car sinon il s'agirait de "partir en Italie pour se promener, et non pas pour apprendre ${ }^{10}$. C'est pour cela que, si l'on veut tracer une ligne évolutive de la pensée architecturale académique madrilène à travers le dessin d'architecture, on s'aperçoit que cette ligne n'existe pas, ou du moins, qu'il s'agit d'une ligne discontinue. Dans l'académie madrilène l'analyse de la production graphique datée entre 1752 et 1808 révèle une grande hésitation à la fois dans la définition du classicisme, qui emprunte parfois au baroque romain, parfois aux modèles palladiens ou même au style de Juan de Herrera et également dans les modes de représentation qui ne semblent pas formalisés : la figuration des ombres n'est pas uniforme, de même 
que l'emploi du lavis ou même de l'usage de la perspective, une définition peu claire qui influence le langage architectural et artistique dans le style et les techniques des dessins. L'architecture académique sur papier à Madrid fut marquée par une longue réflexion autour du classicisme, mais un classicisme nourri indistinctement des références allant de Vitruve à Palladio, de Juan de Herrera au Bernin, des ruines antiques aux modèles de la nouvelle architecture civile internationale.

Par ailleurs, dans la pédagogie madrilène, le dessin d'architecture s'est placé au centre d'un long débat issu de l'incapacité de cette institution à mettre sur pied une véritable méthode d'enseignement. Les professeurs académiques firent écho aux débats autour de l'importance du dessin d'architecture qui avaient vu s'opposer Diderot, Bottari et Milizia ${ }^{11}$. Dans ses Papeles criticos de Arquitectura Diego de Villanueva plaçait le dessin parmi les matières fondamentales dans la formation et la pratique de l'architecte ${ }^{12}$. Cet avis contrastait avec l'assemblée générale de l'académie qui, en août 1760, avait considéré que les concurrents des concours graphiques devaient passer également un examen théorique pour justifier leurs choix techniques et stylistiques. L'assemblée pensait alors que "dans cet art [l'architecture] l'habileté n'est précisément pas prouvée par la beauté du dessin, mais par la connaissance de la raison qui le produit, ainsi que des théories qui règlent et dirigent son travail ${ }^{13}$.

Dans un contexte de lutte contre la tradition corporatiste des architectes retableros (maîtres des retables), dont le caractère baroque et pictural du dessin fut toujours sévèrement contesté par les cercles académiques, certains enseignants ont insisté sur un usage strictement scientifique et empirique du dessin d'architecture (ill. 3). C'est pour cela que José Ortiz y Sanz, théoricien de l'architecture très influent à San Fernando, affirmait que «trop d'importance accordée au dessin pouvait être préjudiciable aux architectes avides d'idées nouvelles et de caprices " ${ }^{14}$. Il est clair que ni le caprice ni la fantaisie n'eurent leur place dans le dessin d'architecture académique en Espagne, pays où, par ailleurs, apparement la vague piranésienne a eu une réception quasiment inexistante ${ }^{15}$. Vers la fin du siècle, une partie des enseignants émirent la critique qu’à force de considérer le dessin comme la preuve absolue des capacités artistiques et professionnelles des architectes, l'académie se contentait juste "de former des académiciens $"{ }^{16}$. Pendant la crise interne

11. Basile Baudez, "L'Europe architecturale du second XVIII ${ }^{\mathrm{e}}$ siècle : analyse des dessins ", Livraisons d'histoire de l'architecture, $\mathrm{n}^{\circ}$ 30, 2015, p. 43-44.

12. Carlos Sambricio, "Diego de Villanueva y los Papeles Críticos de Arquitectura ", Revista de Ideas Estéticas, v. 31, 1973, p. 173.

13. Assemblée Générale du 12 août 1760.

14. "El dar mucha importancia al dibujo puede ser perjudicial para los arquitectos ávidos de ideas nuevas $y$ de caprichos : en esta manía cayó Francesco Borromini ", cité dans Francisco José Leon Tello, María Virginia Sanz Sanz (dir.), Estética y teoría de la arquitectura en los tratados españoles del siglo XVIII, Madrid, CSIC, 1994, p. 615.

15. Voir Carlos Sambricio, "Piranèse et l'Espagne des Lumières ", Piranèse et les Français, actes du colloque international tenu à la Villa Medicis, Roma, Edizioni dell'Elefante, 1978, p. 509-524.

16. "En la academia nos hacemos arquitectos, sino académicos". Discours d'Antonio Varas du 9 octobre 1792 dans ASF 1-18-20. Parmi les diverses réponses des architectes, Juan de Villanueva proposait une réorganisation des salles du dessin (ASF 5-61-4). 


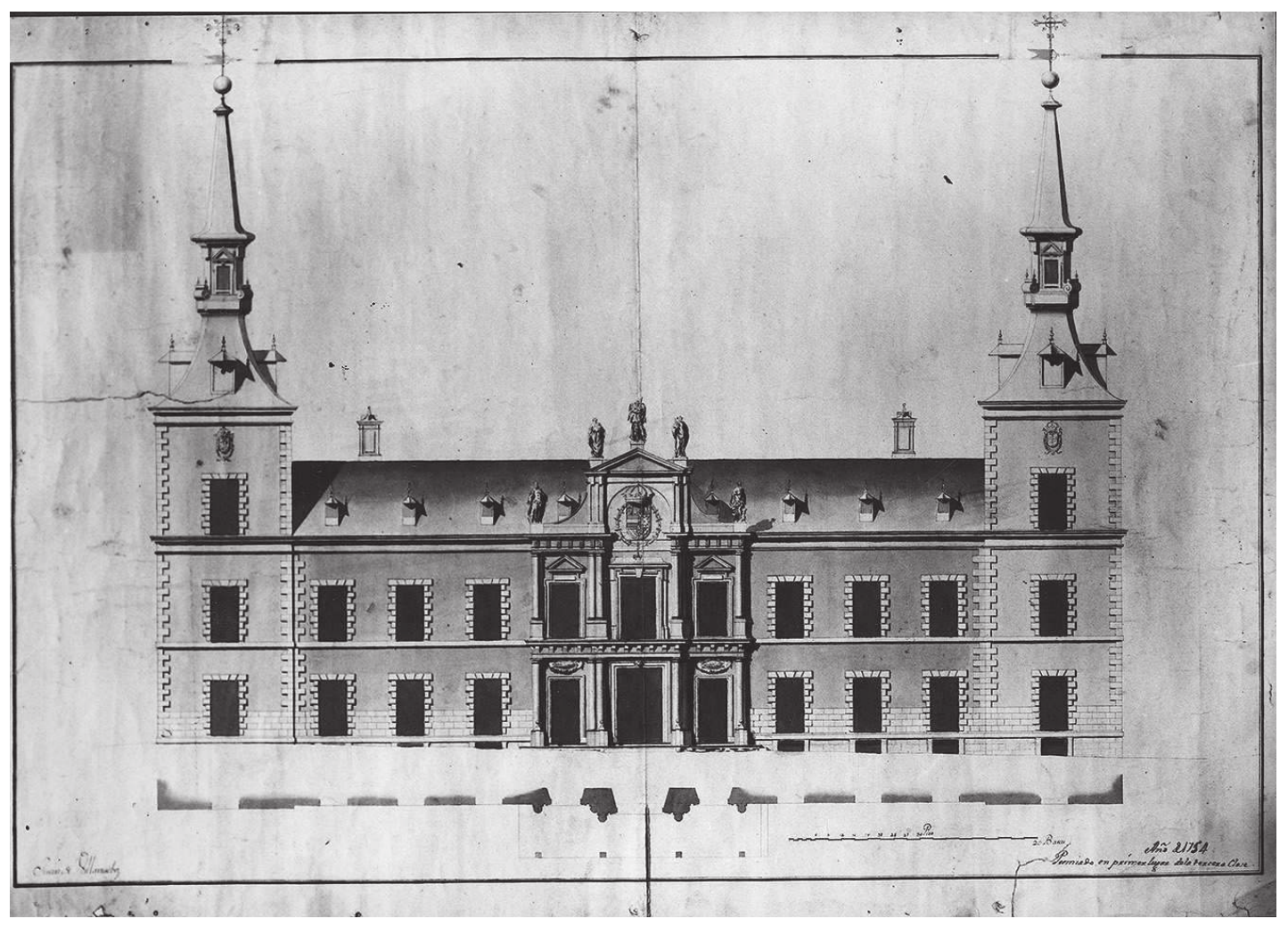

Ill. 3 : Juan de Villanueva, Plan et élévation de la Cárcel de Corte de Madrid, Premier prix pour l'architecture 1754, 63,5 ×93 cm, crayon, encre de Chine et lavis gris. C Madrid, Real Academia de Bellas Artes de San Fernando, A-924.

que connut l'Académie lors de l'année 1792, le mathématicien Antonio Varas décrit également les étudiants académiques comme «des jeunes obsédés à faire quelques petites copies de Scamozzi ou de n'importe quel autre auteur, pour après se mettre à passer des concours " ${ }^{17}$. Par ailleurs, dans ce discours le directeur de la chaire de mathématiques ajoutait que «l'on ne peut pas confondre un grand architecte avec un grand dessinateur: le dessin est sans doute une chose qu'on doit maîtriser, mais ce n'est pas le dessin qui fait l'architecte ${ }^{18}$.

Malgré cette polémique, le dessin d'architecture fut toujours accepté à San Fernando comme un objet artistique en soi. Les élites aristocratiques qui contrôlaient la direction de l'académie le considéraient comme un emblème du bon goût à diffuser sur le territoire national. Cette production graphique était également à leurs yeux l'évidence matérielle des progrès faits par l'institution dans le vaste programme réformiste de l'État bourbonien qui avait conçu l'académie.

Jusqu'au passage entre les deux siècles, le système resta le même : la copie des modèles résistait, mais l'influence des envois de l'étranger favorisait la conception

17. "Los alumnos se obcecan mucho en hacer copias curiositas de Scamozzi o cualquier otro autor, para luego opositar a la Academia ", ASF 1-18-20.

18. Sur la crise des années 1790 à l'académie de Madrid voir José Enrique García Melero (dir.), Renovación, crisis, continuismo : la Real Academia de San Fernando en 1792, Madrid, RABASF, 1992, 158 p. 
de nouveaux dessins d'invention propre, plus personnels et plus modernes tant dans le style comme dans la technique. À partir de 1793, l'académie ouvrit ses salles en été pour faire une exposition publique des meilleurs dessins d'architecture, peintures et sculptures ${ }^{19}$. L'aristocratie et la haute bourgeoisie madrilènes assistent à ces expositions qui célèbrent l'architecture académique comme un pilier fondamental dans la transformation éclairée de la nation. Le dessin d'architecture, admiré comme une œuvre d'art, est donc devenu à la fin du dix-huitième siècle l'un des témoignages artistiques majeurs de ce progrès ${ }^{20}$.

\section{Dessiner l'architecture dans l'académie de Madrid: méthodes, techniques et matériels de la pensée architecturale espagnole sur papier}

Comme dans le reste des institutions académiques européennes, la plupart des dessins conservés à Madrid ont été créés à partir de programmes très spécifiques avant d'être soumis au jugement d'un jury qui, à l'Académie de San Fernando n'a pas favorisé la recherche de l'innovation ou de l'originalité ${ }^{21}$. Ce sont généralement des dessins analytiques, faits pour développer un apprentissage ou atteindre une reconnaissance institutionnelle. En conséquence, ce ne sont pas des manifestations libres de la pensée architecturale individuelle de leur auteur, et ils ne représentent pas forcement toute sa culture artistique ou ses connaissances techniques.

Cela dit, on peut quand même percevoir une évolution thématique, stylistique et technique propre et effective dans la manière de représenter l'architecture à l'académie de San Fernando. Les exemples les plus remarquables appartiennent aux rendus des concours académiques et aux envois de Rome et de Paris. Sauf la prueba de repente, qui laissait juste deux heures de travail aux concurrents, le reste de dessins rendus aux jurys sont le fruit de longues et méticuleuses heures de travail afin de se conformer aux strictes exigences des académiciens (ill. 4). À Madrid le dessin "a sentimiento" (spontané, rapide), ne rentrait pas (au moins de manière officielle) dans la pédagogie académique, chose curieuse pour un pays qui a accueilli de magnifiques maîtres de ce genre de dessins, comme Filippo Juvarra, mort à Madrid en 1736, et dont les élèves comme Ventura Rodriguez, magistral dessinateur luimême, n’ont pas perpétué cette manière de dessiner dans leurs cours. Bien que les programmes des concours ne donnent pas d'indications sur les matériaux ou les

19. ASF 1-55-2, "Euvres d'art des professeurs des Beaux-Arts exposées au public pendant les mois d'été entre 1793 et 1808. "Cet inventaire cite notamment de nombreux dessins des grands architectes académiciens de la période perdus certainement à cause de cette surexposition.

20. Voir notamment le rapport envoyé en 1795 par le conseiller de l'Académie à Isidoro Bosarte sur les "progrès faits par les arts de l'institution, et notamment l'architecture " dans lequel il affirmait " avoir entendu personnellement les éloges faits à un de ses disciples en Italie " (ASF 1-28-1).

21. L'ensemble de ces jugements où on peut constater un fort écart entre les remarques de l'assemblée académique et les formes nouvelles dont l'usage est encouragé dans d'autres centres européens se trouve dans les actes de la Commission d'architecture et des séances académiques, tous disponibles en ligne sur http://www.cervantesvirtual.com/portales/bellas_artes_san_fernando/archivo/. 


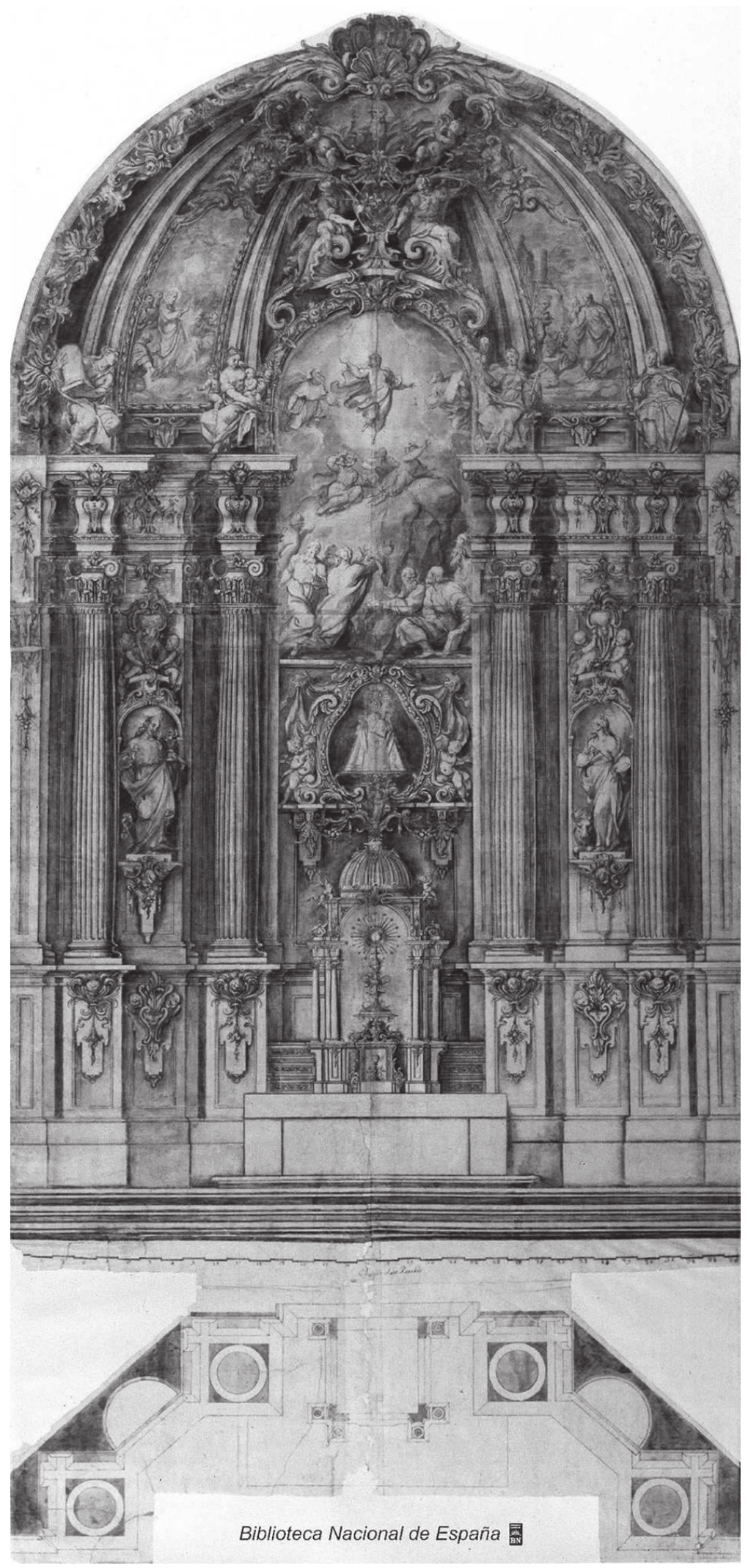

Ill. 4: Teodoro Ardemans, Projet pour un retable de la transfiguration (plan et élévation), c. 1730, $153,2 \times 72,5 \mathrm{~cm}$, crayon, encre de Chine, lavis gris, brun et bleu. (C) Madrid, BNE, Dib/15/85/82. 
techniques, on insiste toujours sur le type de papier à utiliser (papier de Hollande), et surtout sur le caractère géométral des objets architecturaux à rendre. Entre 1752 et 1800 se développe un type de dessin d'architecture académique très codifié qui, sauf quelques exceptions, suit des caractéristiques peu variables. En général, tous ces rendus sont d'un format moyen, dont la proportion de l'objet est quasiment toujours donnée par une échelle en pieds castellans ${ }^{22}$. La triade vitruvienne - plan, élévation et coupe - est toujours respectée, souvent sur une même feuille. Par ailleurs, une autre feuille est parfois ajoutée avec des détails du bâtiment, ainsi que des textes explicatifs très sommaires, loin des fioritures typiques des textes des dessins des concours romains contemporains. Jusqu'à la fin du siècle, le dessin d'architecture à San Fernando se caractérise par une proportion à la monochromie. Les jeunes élèves utilisent surtout le crayon, l'encre de Chine et le lavis gris, dont les effets rapprochent beaucoup ces dessins des gravures et des estampes qu'ils copiaient dès leur entrée à l'académie. Le lavis rose n'apparaît dans les coupes que vers les années 1780 , et on conserve très peu de rendus dont la variété de couleurs des lavis utilisés soit remarquable ${ }^{23}$. Le caractère mesuré et sobre de ces exercices académiques s'accompagne d'un usage des ombres très discret, marqué dès les années 1760 par une utilisation généralisée des ombres à $45^{\circ}$. En général, la production académique de ces années rend peu compte des influences de cette révolution opérée dans l'architecture française contemporaine dont Krafft parlera quelques années plus tard ${ }^{24}$. À Madrid, le débat autour de l'architecte-peintre n'a quasiment jamais été abordé. Les rares occasions où la question s'est vaguement posée relevaient toujours de la condamnation de la manière pittoresque des architectes retableros formés dans la tradition corporatiste que l'académie voulait éteindre. On peut tout à fait imaginer que les gravures des Grands Prix parisiens arrivent à Madrid à partir des années 1790, mais celles-ci, dépourvues des effets spectaculaires des dessins originels, ont eu une influence nulle sur la technique madrilène ${ }^{25}$. Par ailleurs, et malgré leur enseignement permanent à l'académie, les concurrents de San Fernando utilisent très rarement les perspectives; les quelques exemples que nous en avons sont d'une qualité médiocre (ill. 5). À la différence de Paris, les élèves de San Fernando n'ont introduit dans leurs dessins que très tardivement, dans la première

22. Rappelons que, concernant la taille des dessins, par exemple, dans le $10^{\mathrm{e}}$ point de l'instruction des pensionnaires de 1791 on insistait sur «la taille adéquate et homogène [des envois] pour que l'on puisse constituer une collection solide et décente" (ASF 1-49-6).

23. Citons par exemple le "Palais épiscopal» de Pedro Arnal (1763), le "Templo de la Inmortalidad" d'Antonio López de Losada (1772), ou la "Casa de Campo» de Juan Antonio Cuervo (1784), dessins dont l'usage des lavis reste très contradictoire et sans suivre une méthode établie.

24. Katia Frey, "La figuration graphique de l'architecture néo-classique et le rôle de la gravure au trait dans les recueils de Jean-Charles Krafft ", Klassizismen und Kosmopolitismus : programm oder problem? Austausch in Kunst und Kunstheorie im 8. Jahrhundert, Pascal Griener, Kornelia Imesch dir, Zürich, Schweizerisches Institut für Kunstwissenschaft, 2004, p. 221.

25. Concernant la reproduction des Grands Prix, dont la diffusion influence les Espagnols à Rome autour du concours clementinien de 1795, voir Helen Rosenau, "The engravings of the Grand Prix of the French Academy of Architecture ", Architectural History, vol. 3, 1960, p. 17-180. 


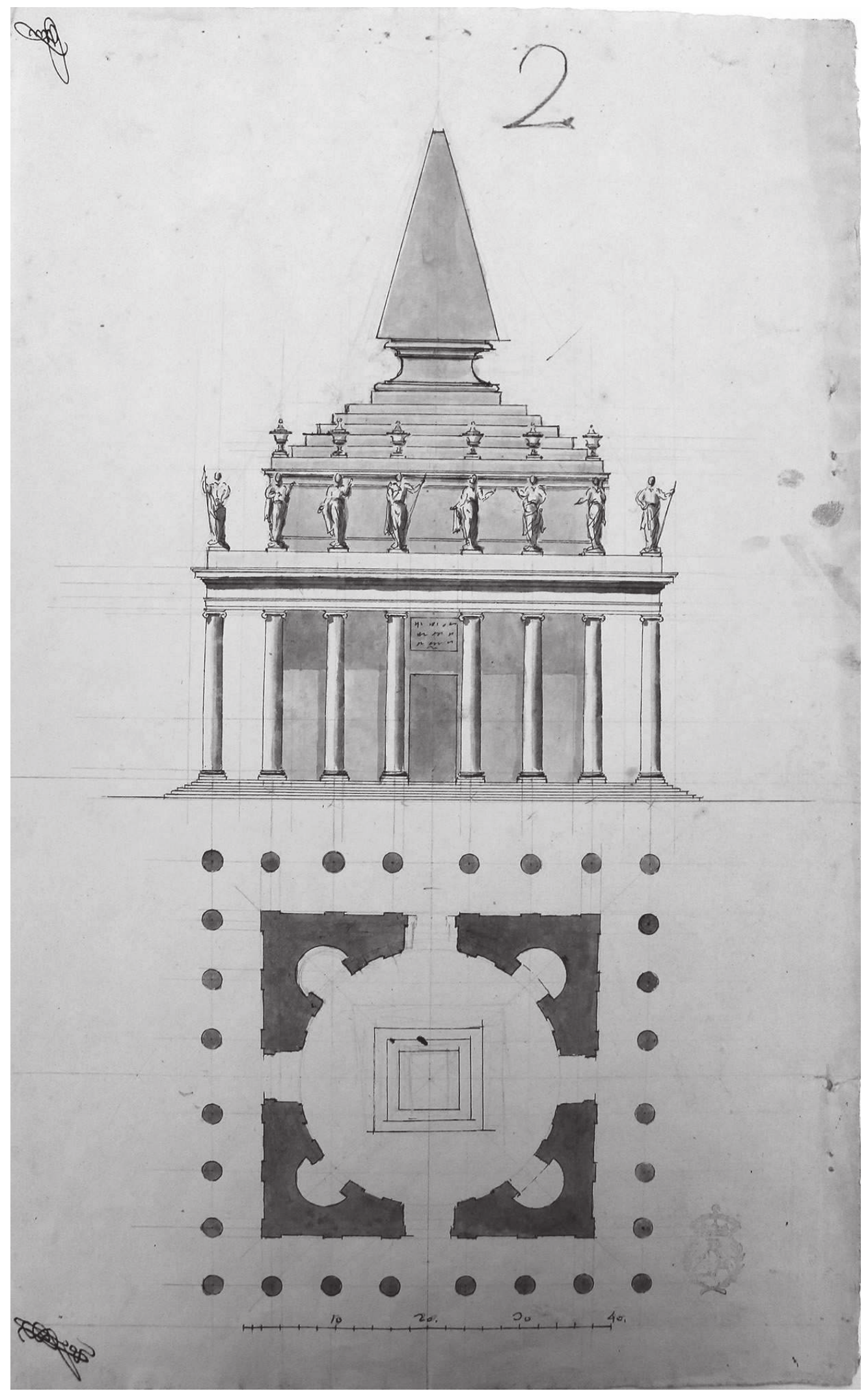

Ill. 5: Guillermo Casanova, Un mausolée d'après les anciens (plan et élévation), Prueba de repente pour la deuxième classe d'architecture de $1778,49 \times 30,3 \mathrm{~cm}$, crayon, encre de Chine, lavis gris. (C) Madrid, Real Academia de Bellas Artes de San Fernando, A-4925. 


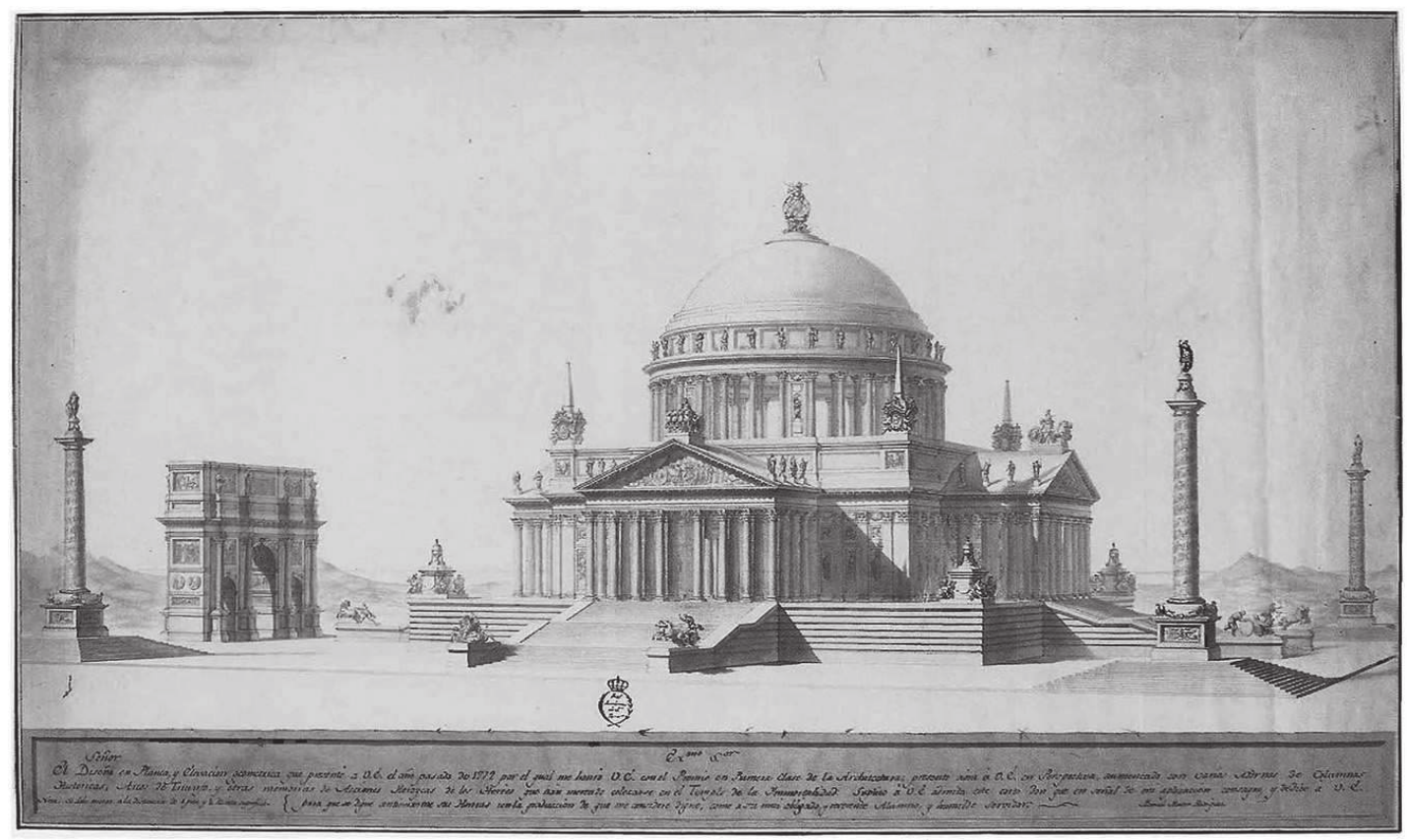

Ill. 6: Manuel Martín Rodríguez, Temple de l'honneur et de l'immortalité (vue perspective), Prix de $2^{\mathrm{e}}$ classe $1772,58,2 \times 97,5 \mathrm{~cm}$, crayon, encre de Chine, lavis gris et brun. (C) Madrid, Real Academia de Bellas Artes de San Fernando, A-4465.

décennie du XIX ${ }^{\mathrm{e}}$ siècle les cieux nuageux, les contrastes dramatiques de lumière, ou les fonds paysagers.

Cependant, dans les années 1790, on a vu arriver à Madrid d'importantes nouveautés en matière de dessin d'architecture, toujours liées à l'activité des pensionnaires espagnols à l'étranger. Ces pensionnaires passaient par Paris et intègraient les cénacles intellectuels modernes de la Rome internationale, en concevant des dessins dont l'influence se cristallisa plus tard à Madrid, et ouvrit de nouvelles perspectives graphiques dans les premières années du siècle suivant (ill. 6) ${ }^{26}$. Il s'agit, comme l'a remarqué Delfín Rodríguez, des années « les plus intéressantes et les moins étudiées de l'architecture espagnole " ${ }^{27}$. Les envois de Juan Gómez (élève de JulienDavid Leroy à Paris), Silvestre Pérez, Jorge Durán, Isidro González Velázquez, ou Eusebio María de Ibarreche marquent, dans les années années 1790, un point d'inflexion dans l'histoire du dessin d'architecture espagnol. Parmi ces dessins, ceux qu'Ibarreche envoya en 1793 à l'académie constituent l'un des exemples les plus remarquables de l'évolution de la technique du dessin d'architecture madrilène

26. Soulignons qu'entre les versions romaine (ASL-0909 - ASL 0912) et madrilène (ASF A-4858 A-4861) de la chapelle sépulcrale de Jorge Durán, cette dernière présente une richesse plus grande dans l'usage des lavis et des effets de lumière. Ces différences furent certainement introduites par Durán lors de l'exposition publique des prix d'architecture à Rome sous l'influence des dessins de Giovanni Campana, ce qui prouve l'importance de ces expositions publiques pour l'apprentissage des jeunes architectes.

27. Delfín Rodríguez, Hacia una nueva idea de arquitectura, op. cit., p. 27. 


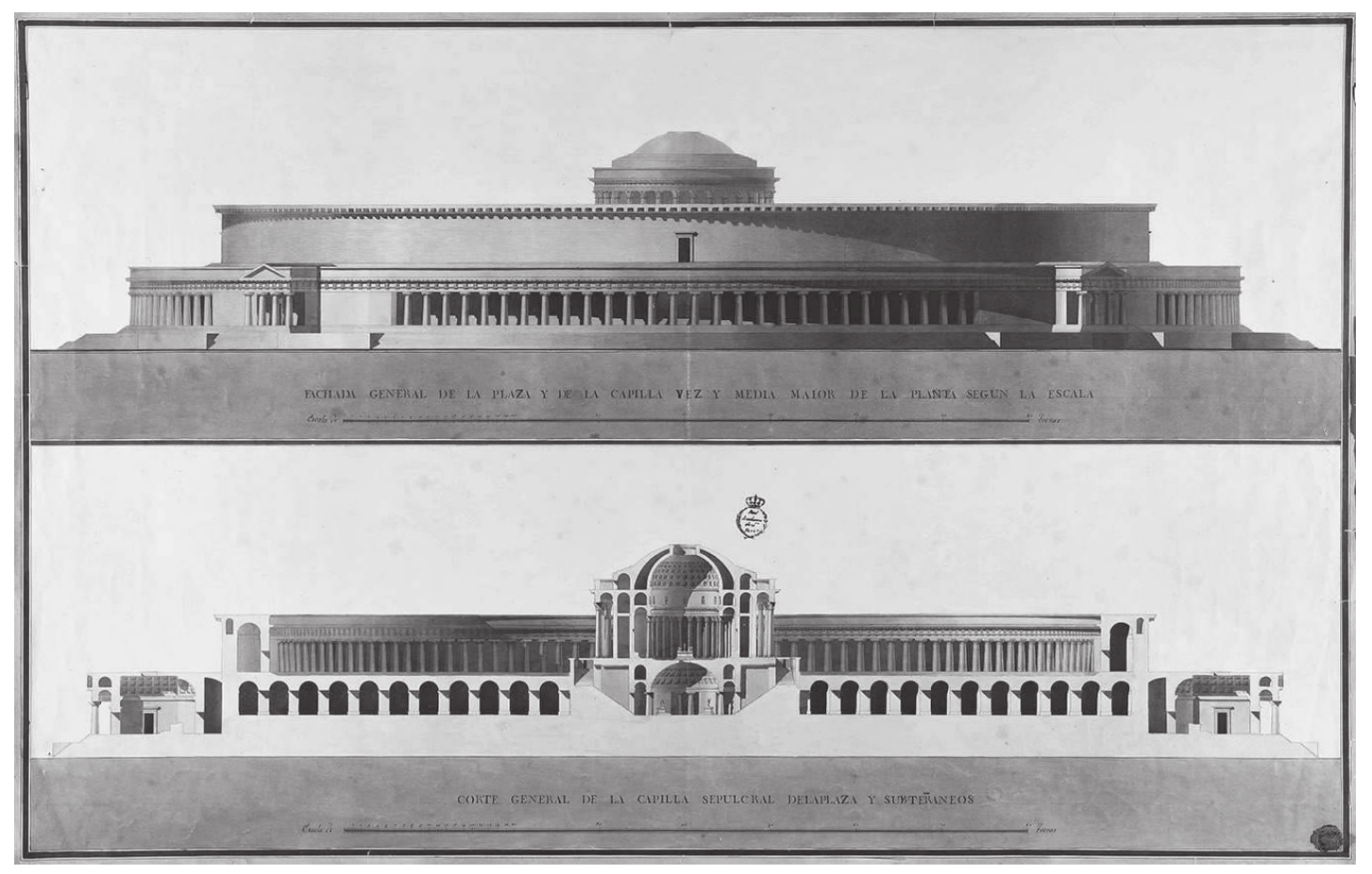

Ill. 7 : Jorge Durán, Chapelle sépulcrale (élévation et coupe longitudinale), copie du projet originel du Premier Prix ex-aequo du Concorso Clemetino 1795, $63 \times 99 \mathrm{~cm}$, encre de Chine, lavis gris, brun, rose, bleu. (C) Madrid, Real Academia de Bellas Artes de San Fernando, A-4858.

(ill. 7) ${ }^{28}$. Sur les cinq feuilles de ses bains publics, le pensionnaire employa, de manière inédite, une grande quantité de lavis de couleurs servant à différencier les parties du projet, les surfaces, et même les différents matériaux. À côté de ce riche éventail de couleurs, il a également introduit des effets d'ombres plus marqués, en renfonçant le côté pittoresque des dessins par les détails de l'eau et de la végétation qui nuancent l'ensemble. Par ailleurs, le plan général montre également un usage très romain du trompe-l'œil pour les cartouches contenant les longs textes explicatifs. Ces dessins prouvent, comme ceux de Silvestre Pérez, la connaissance des Espagnols de la technique de l'acquatinta, en vogue dans la Rome contemporaine. Au regard de ces évidences jusqu'ici ignorées, la forte connexion entre les pensionnaires de San Fernando et les membres de l'Accademia della Pace, dont la fameuse Raccolta di proggetti architettonici a promu la popularité de l'acquatinta parmi les architectes de la Rome internationale, doit être reconsidérée ${ }^{29}$. On sait que Silvestre Pérez et Jorge Durán étaient des pacisti, mais Ibarreche et les autres architectes du cercle de l'ambassadeur espagnol Nicolás d'Azara ont probablement dû aussi fré-

28. Architecte encore très méconnu, il travailla sous le mécénat du duc d'Albe, et reçut une pension extraordinaire de Charles IV pour aller à Rome, où il dessina ses Bains publics en 1793 (ASF A-1957 à A-1961), un projet qui lui valut la nomination d'académicien de mérite en 1794.

29. Sur cette académie privée qui dispensait un enseignement très avant-gardiste en dehors du discours officiel de San Luca nous renvoyons à l'étude pionnière de Susanna Pasquali, "Contributo alla conoscenza della cultura architettonica a Roma alla fine del 700 : Felice Giani e l'Accademia della Pace ", Quaderni del dipartimento patrimonio architettonico e urbanistico, $\mathrm{n}^{\circ}$ 3, 1992, p. 76-87. 
quenter les séances de ce cénacle dont la modernité de l'architecture proposée, ainsi que les nouveautés dans sa traduction graphique, étaient très renommées dans la Rome de cette époque.

Cependant, il faut souligner que ces nouveautés dans la représentation de l'architecture ne concernent souvent que les dessins privés de ces jeunes pensionnaires. Pendant les années 1790 où l'on a vécu la plus grande transformation dans l'architecture dessinée espagnole, s'opère également une dissociation sans précédents entre une production pour l'académie, et une autre faite par les jeunes pensionnaires pour le pur plaisir d'inventer des images architecturales. Cette dernière production s'est notamment développée sous l'influence des échanges internationaux produits dans des milieux artistiques non officiels. Dans une décennie de profonde crise pour l'académie madrilène, il semblerait que les pensionnaires à Rome aient conçu une architecture "officielle » juste pour contenter le jury académique dont leurs pensions dépendaient. Ces dessins, quasiment toujours des relevés

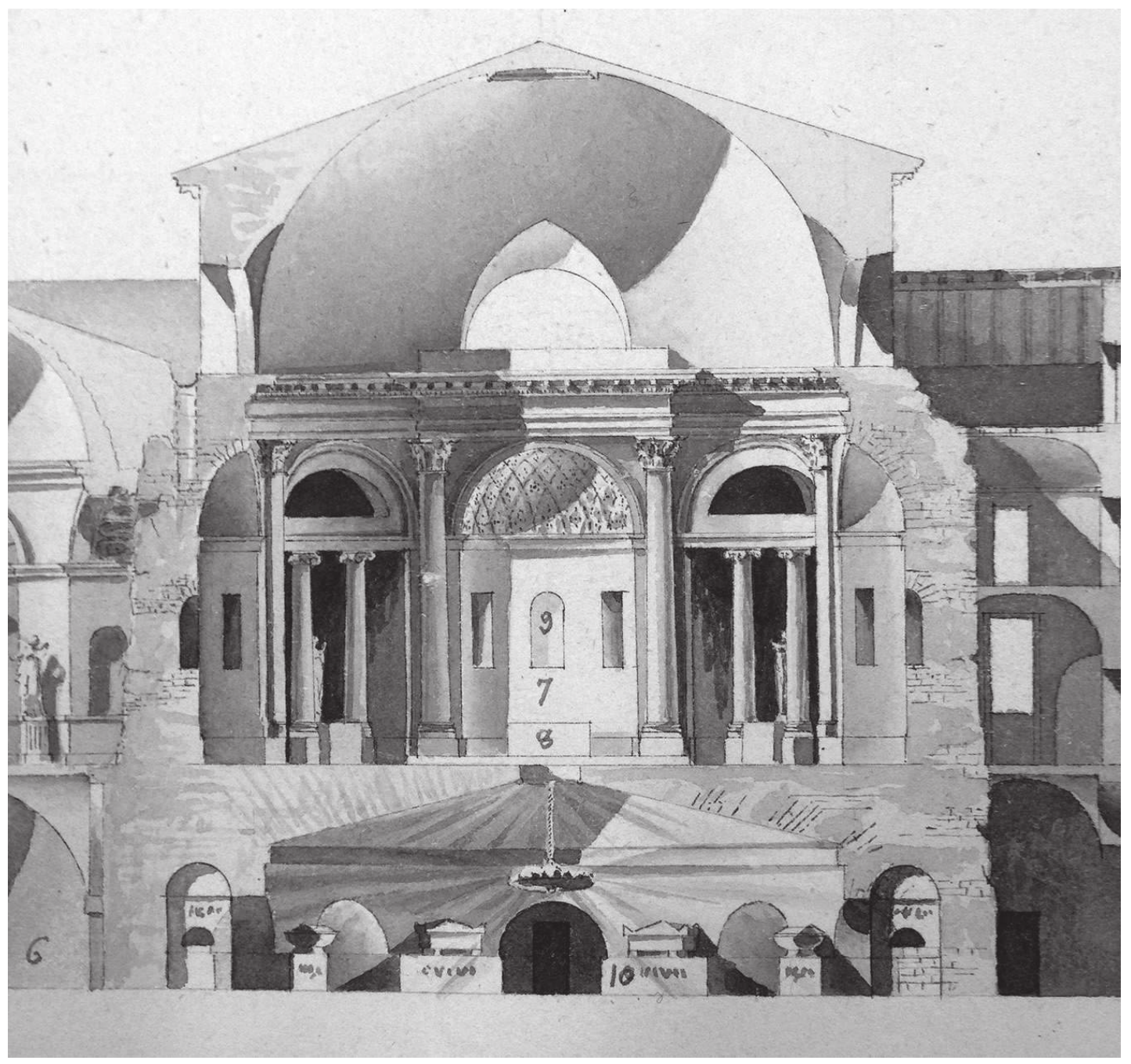

Ill. 8 : Eusebio María de Ibarreche, Maison de bains publics (détail de la coupe longitudinale), 1794, $67 \times 99,5 \mathrm{~cm}$, encre de Chine et lavis rose, brun, orange et gris. (C) Madrid, Real Academia de Bellas Artes de San Fernando, A-1959. 


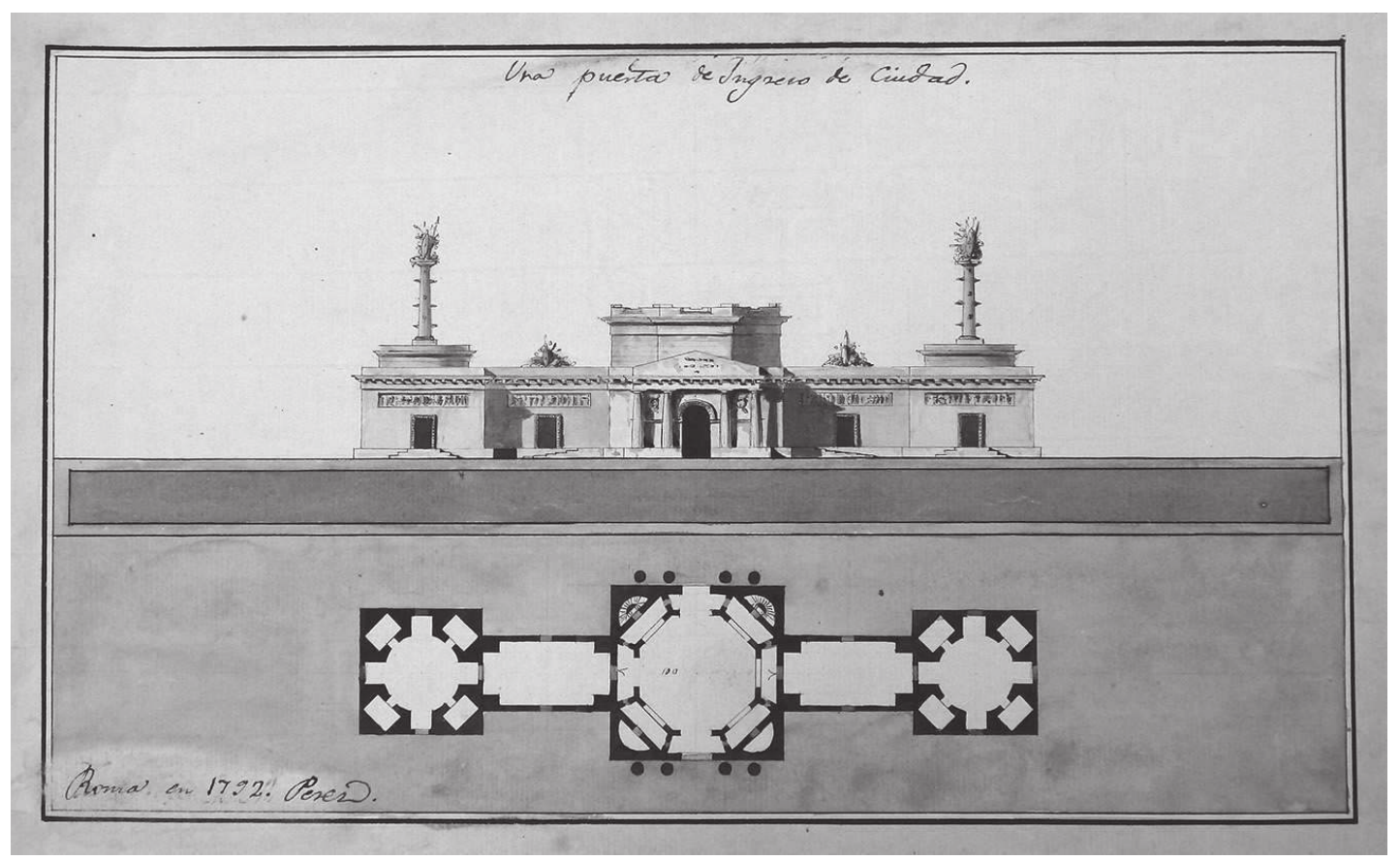

Ill. 9 : Silvestre Pérez, Porte d'entrée à une ville, c. 1792, $25 \times 34 \mathrm{~cm}$, encre de Chine, lavis brun, gris, rouge. (C) Madrid, Real Academia de Bellas Artes de San Fernando, A-3821.

de ruines antiques parfaitement dessinés et lavés, ont reçu des critiques très positives bien qu'ils n'apportaient strictement rien de nouveau au discours théorique de l'académie. Néanmoins, à côté de ces envois il y avait les ruines pittoresques faites à l'aquarelle par Isidro González, ou les dessins de petit format sur lesquels Silvestre Pérez apprenait la nouvelle architecture civile en utilisant de manière très libre les ombres et les lavis de couleurs (ill. 8) ${ }^{30}$. La comparaison de cette double production montre clairement le décalage qu'il y avait à cette époque entre l'académie de San Fernando et ses jeunes émissaires. L'étude de ces dessins privés des pensionnaires de l'académie de Madrid révèle, au-delà de la qualité de leurs envois officiels, la vérité sur la compréhension - la connaissance ? - espagnole de l'architecture contemporaine. Lors de leur retour, ces jeunes ont occupé progressivement des postes dans l'enseignement académique, en formant des élèves sous l'influence des dessins faits dans les années 1790 à Rome, considérés plus que jamais comme des modèles à imiter. Entre 1800 et 1808, on peut considérer que le dessin d'architecture académique madrilène arrive au sommet de sa qualité. Les principaux exemples de cette période montrent comment les typologies se diversifient, la composition s'enrichit, la taille et l'échelle augmentent, l'usage des lavis de couleurs se généralise, et les effets d'ombre et de lumière se nuancent de manière très judicieuse. À l'aube

30. L'ensemble de ces dessins, qui sont restés avec leurs auteurs jusqu'à la fin de leurs vies, se trouve à la Bibliothèque nationale espagnole. Sur Silvestre Pérez voir son recueil de Trazas y rasguños BNE Dib/14/27. L'ensemble de dessins d'Isidro González Velázquez est reproduit avec leurs caractéristiques techniques dans Pedro Moleón (dir.), Isidro Gonzázlez Velázquez: arquitecto del Madrid Fernandino, Madrid, Ayuntamiento de Madrid, 2009, 577 p. 


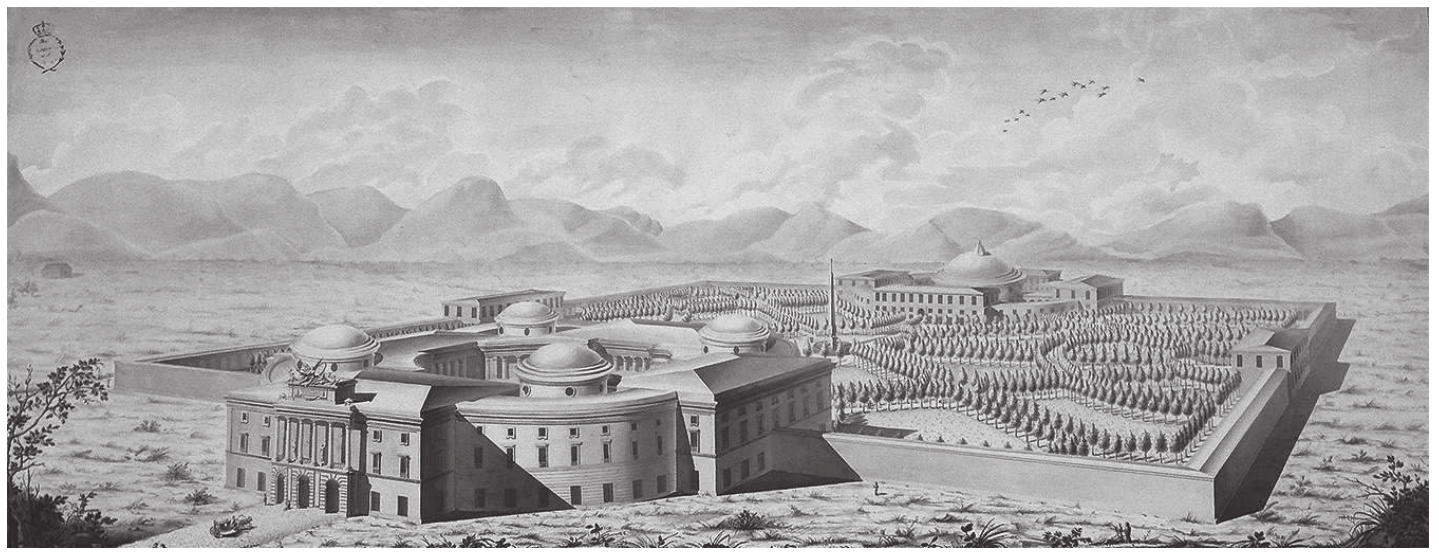

Ill. 10 : Joaquín de San Martín, Gymnase pour la grandeur d'Espagne (détail de la vue générale), 1804, $112 \times 67 \mathrm{~cm}$, encre de Chine, lavis gris et brun, aquarelle. (C) Madrid, Real Academia de Bellas Artes de San Fernando, A-476.

du XIX ${ }^{\mathrm{e}}$ siècle l'architecture sur papier madrilène accepte aussi l'usage à grande échelle des éléments pittoresques et paysagers (ill. 9). On est donc finalement arrivé à cette nouvelle idée d'architecture que l'académie de Beaux-Arts de Saint-Ferdinand avait tellement cherchée au fil des ans.

Adrián ALMOGUERA

Université Paris-Sorbonne, Centre André Chastel 